data collection and a larger computer for analysis, for example, the TRS- 80 Model 100/TRS-80 Model $4^{8}$ or Epson PX-8/BBC Model B ${ }^{9}$. Other systems use the IBM PC as a host computer with, for example, a NEC PC-8201A, PC-8300 $0^{10}$, or Epson HX $-20^{11}$ as an event recorder. Data-collection software has even been developed for HP41 and HP71 pocketcomputers ${ }^{12}$. However, with the current short lifetime of computer models, specific programs written for a particular type of computer run the risk of soon becoming obsolete.

As most behavioural scientists lack programming expertise, there is a need for general-purpose event recording and data analysis software. Such software should (1) be very flexible with regard to hardware and experimental design, (2) be intuitively understandable and require no programming by the user, (3) be robust against user errors, and (4) produce data in a format compatible with commercially available analysis software.

The Observer (Fig. 1), a recently developed software package for observational research ${ }^{13}$, represents an attempt in this direction. This program runs on any IBMtype PC. The user can configure the event recorder accurately to many different experimental designs. With the information entered by the user, the PC can be used directly as an event recorder. In addition, four types of non-IBM compatible portable computers are supported as event recorders: TRS-80 Model 100, Tandy 102, Olivetti M10, and Epson PX8. For these computers, The Observer generates event-recording programs specifically adapted to the machine and experimental design chosen by the user. As the programs contain only code essential to the current task, they remain compact and occupy a minimum amount of memory in the portable computer. The program transfers generated programs to the portable event recorder and retrieves collected data files. The Observer also analyses observational data and provides the user with statistics in printed reports.

The trend in software is towards computer-user interaction at an increasingly higher level. Ideally, the user should merely have to specify what the problem is, not how it should be solved. The development of such high-level software opens new possibilities for computer-aided behavioural research. With the arrival of computer programs that do the programming, the behavioural scientist can concentrate better on the actual research.

Lucas P. J. J. Noldus is at the Dept. of Entomology, Agricultural Univ. Wageningen. The Netherlands. Erik L. H. M. van de Loo is at the Dept. of Clinical and Health Psychology, Univ. of Leiden, and the Inst. for Human Resource Management, The Netherlands. Paul H. A. Timmers is at Philips Data Systems BV, Apeldoorn. The Netherlands. For more information, fill in the reader service number 100.

\title{
New tools for neuroscience
}

Exhibits at next week's American Society for Neuroscience Annual Meeting in Phoenix, Arizona include eicosanoid ElA kits and an activity monitor.

THREe new eicosanoid enzyme immunoassay kits for the measurement of prostaglandin $\mathrm{E}_{2}$, thromboxane $\mathrm{B}_{2}$ and 6-ketoprostaglandin $F_{1 \alpha}$ are available from Advanced Magnetics, Inc. (Reader Service No. 101). These solution-phase assays are based on the competition of variable amounts of analyte with a fixed amount of alkaline phosphatase-labelled analyte for a limited number of rabbit antibody binding sites. The addition of magnetic goat anti-rabbit antibody, followed by magnetic separation or centrifugation, separates antibody-bound from unbound analyte. The resulting pellet is reacted with para-nitrophenyl phosphate substrate and the absorbance read at $405 \mathrm{~nm}$ : sample concentration is determined from a standard curve. AMI says, the prostaglandin $\mathrm{E}_{2}$, thromboxane $B_{2}$ and 6-keto-prostaglandin $F_{1 \alpha}$ can detect $0.13 \mathrm{pg}, 4.0 \mathrm{pg}$ and $0.12 \mathrm{pg}$ per $0.1 \mathrm{ml}$ sample, respectively. The $\$ 295$ (US) kits contain enough reagents for 100 assay tubes, including standard curves, says AMI, who will be in booth 425 .

\section{Data acquisition}

HVS Image will be exhibiting, in booth 2011, their VP118 Super Track Unit activity monitor for monitoring the behavioural activity of animals, particularly rodents (Reader Service No. 102). The VP118 receives input from a video camera positioned over an open field, water or radial maze. Positional information from the image is extracted by the VP118 and relayed to the user's IBM PC/ XT/AT. Super-Track software analyses data for path length, positional preference, and percentage time spent moving. The system can track two animals together, which is useful for studying social interaction, and can also measure rota-

1. Torgerson, L. Behav. Res. Meth. Instrum. 9, 405-406 1977).

Stephenson, G.R. \& Roberts, T.W. Behav. Res. Meth Instrum. 9, 434-441 (1977).

3. Paggeot, B., Kvale, S., Mace, F.C. \& Sharkey, R.W. J. appl. Behav. Anal. 21, 429 (1988)

. Krauss, R.M., Morrel-Samuels, P. \& Hochberg, J. Behav Res. Meth. Instrum. Comp. 20, 37-40 (1988).

5. Flowers, J.H. Behav. Res. Meth. Instrum. 14, 241-249 (1982)

6. Deni, R. Perc. Mot. Skills 65, 398 (1987).

7. EventLog (Conduit, Univ. of lowa, lowa City, lowa)

8. Deni, R., Szijarto, K., Eisler, A. \& Fantauzzo, C. Behav. Res. Meth. Instrum. Comp. 15, 616 (1983).

9. Unwin, D.M. \& Martin, P. Behaviour 101, 87-100 (1987)

10. Juniper Gardens Children's Project, Kansas City, Kansas: $S \& K$ Computer Products, Toronto, Ontario.

11. PCS (Communitech International, Wheaton, Illinois)

12. Whiten, A. \& Barton, R.A. Trends Ecol. Evol. 3, 146-148 (1988)

13. Noldus, L.P.J.J. Abstr. 1st Eur. Congr. Psychol. (Amsterdam), 567 (1989), and Abstr. 21st int. Ethol. Conf (Utrecht), 126 (1989). tional activity. In quad mode, the VP118 can track up to four trials at once.

World Precision Instruments, Inc., exhibiting in booths 923-927, will be showing off the PolyGraf/8 data aquisition system, which provides real-time 'gapless' multichannel data collection, display and storage in the form of a data logger or scrolling strip chart recorder (Reader Service No. 103). The $\$ 1,695$ (US) PolyGraf/ 8 system includes a plug-in data acquisition card for a MacII, IIx or IIcx, providing eight channels of single-sided bipolar input, a BNC signal manifold, userfriendly software, and cable assembly. Special features include storage of data files and data display during aquisition and in playback mode, adjustable forward and reverse scrolling speed for visual tracking in playback mode, and multiple channel overlay. With PolyGraf/8, the user can 'mark' data points and take snapshots of points of interest for incorporation into word processing documents.

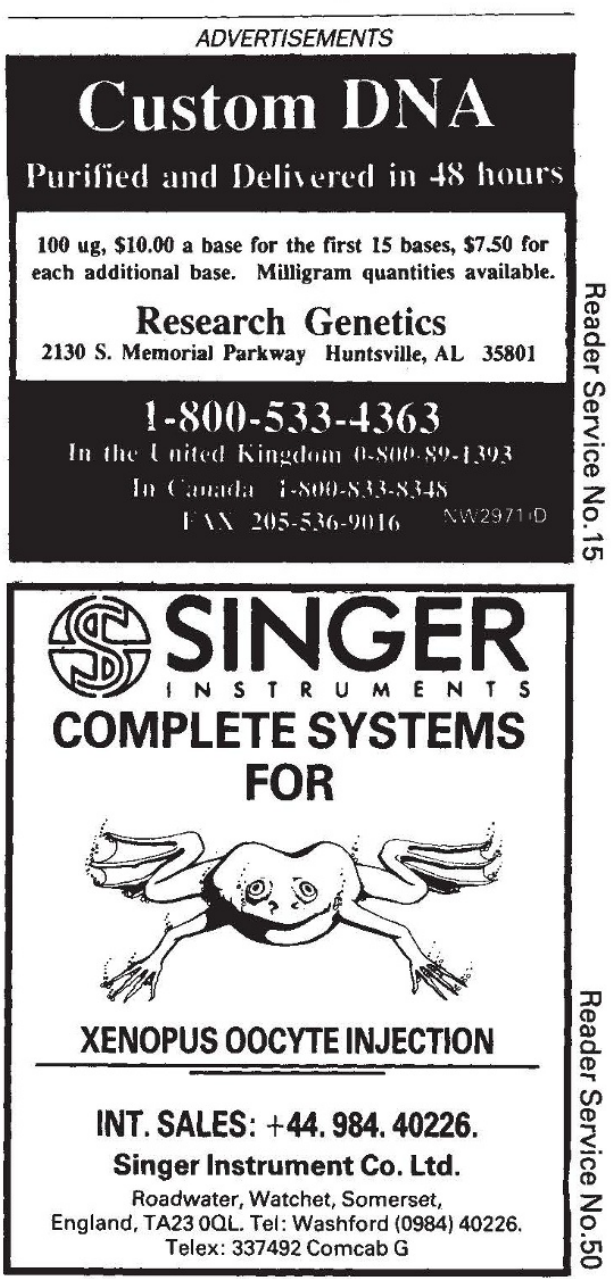

NATURE · VOL $341 \cdot 26$ OCTOBER 1989 